\title{
Latent Class Analysis of Mental Health in Middle Childhood: Evidence for the Dual-Factor Model
}

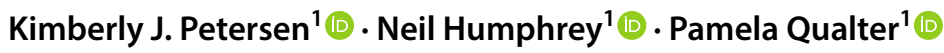

Published online: 25 July 2020

(c) The Author(s) 2020

\begin{abstract}
Mental health is complex, comprising both mental distress and well-being. This study used latent class analysis to identify common combinations of mental distress and well-being ('mental health classes') among schoolchildren aged 8-9 years $(N=3340)$. Thirteen items, measuring a range of conduct problems, emotional symptoms, and subjective well-being, were included in the analysis. Four mental health classes were identified: (1) complete mental health $(n=1895,57 \%)$, (2) vulnerable $(n=434,13 \%)$, (3) emotional symptoms but content $(n=606,18 \%)$, and (4) conduct problems but content $(n=404,12 \%)$. The classes were reliably identified across different datasets, and for males and females. Differential relations with covariates indicated that mental health classes were distinct and externally valid. The results supported the dual-factor model of mental health, suggesting that mental distress and subjective well-being are separate continua. Three of the four possible combinations of high and low distress and subjective well-being posited by the dual-factor model were found using this inductive statistical method. Importantly, our analysis also revealed two 'symptomatic but content' groups, differentiated by symptom domain (internalising/externalising). The covariate analyses between mental health classes and sociodemographic factors, prior academic attainment, school connectedness, and peer support, indicated that there are nuanced relations between those variables and particular constellations of mental distress and well-being. As one of the few dual-factor studies to focus on middle childhood, the current study adds important new evidence that contributes to our understanding of the complexities of mental health among schoolchildren.
\end{abstract}

Keywords Dual-factor model $\cdot$ Mental health $\cdot$ Subjective well-being $\cdot$ Latent class analysis

\section{Introduction}

It is increasingly recognised that mental health is more than simply the presence or absence of symptoms. Researchers in the positive psychology tradition have long argued for a move away from traditional disease-based accounts (Seligman \& Csikszentmihalyi, 2000), placing greater emphasis on subjective well-being, which refers to life satisfaction and overall levels of positive affect (Diener, 1984). Consideration of subjective well-being alongside symptoms helps to

Electronic supplementary material The online version of this article (https://doi.org/10.1007/s12310-020-09384-9) contains supplementary material, which is available to authorized users.

Kimberly J. Petersen

kimberly.petersen@manchester.ac.uk

1 Manchester Institute of Education, The University of Manchester, Manchester M13 9PL, UK develop a more complete understanding of mental health (Antaramian, Huebner, Hills, \& Valois, 2010; Keyes, 2013). Furthermore, it allows the investigation of those who are yet to show symptoms and those that are flourishing. This is particularly important when investigating mental health in children in the general population (Huebner \& Hills, 2011). With the recent focus on preventing mental health problems in schoolchildren in the United Kingdom (UK; Department of Health and Department for Education, 2015, 2017) and more widely (World Health Organisation, 2012) a more complete and nuanced understanding of mental health in middle childhood is required.

\section{The Dual-Factor Model of Mental Health}

The dual-factor model suggests that subjective well-being and symptoms of distress form distinct aspects of mental health (Greenspoon \& Saklofske, 2001). Therefore, at least four mental health 'types' are possible: (1) low symptoms 
and high well-being (i.e. 'complete mental health'), (2) low symptoms and low well-being (i.e. 'vulnerable'), (3) high symptoms and high well-being (i.e. 'symptomatic but content'), and (4) high symptoms and low well-being (i.e. 'troubled') (Suldo \& Shaffer, 2008; Suldo, Thalji, \& Ferron, 2011; Suldo, Thalji-Raitano, Kiefer, \& Ferron, 2016; Thalji, 2012). If mental health symptoms are investigated alone, both the 'vulnerable' and 'symptomatic but content' groups cannot be identified. Furthermore, membership of the different mental health groups has been shown to predict developmental outcomes in young people. Those who are 'symptomatic but content' show better academic outcomes, have higher perceived social support, and better physical health, compared to 'troubled' youths, even though both groups show symptoms of distress. In addition, those that are 'vulnerable' have poorer social relationships and worse academic outcomes compared to those with 'complete mental health', despite both having few symptoms (Suldo et al., 2011, 2016). This suggests that mental distress and subjective well-being are distinct continuums, and that the combination of distress and subjective well-being is important for young people's development.

\section{Traditional Methods of Studying the Dual-Factor Model}

Studies investigating the dual-factor model of mental health have typically identified subgroups by using clinical or sample-based cut-offs to dichotomise mental distress and well-being scores as high or low, and then cross-tabulating those to form the four groups noted above (e.g. Antaramian et al., 2010; Lyons, Huebner, Hills, \& Shinkareva, 2012; Suldo \& Shaffer, 2008). That method can indicate a dissociation between mental distress and well-being, but it has significant limitations. First, the use of imposed cutoff points means there may be little differentiation between groups: individuals with similar scores that fall either side of the cut-off would be placed in different groups. Further, there could be substantial variability within groups: a large range of scores may be included within a particular group (Baillargeon, Tremblay, \& Willms, 1999; Dowdy \& Kamphaus, 2007; Kim, Dowdy, Furlong, \& You, 2017). That is particularly problematic when looking at relations between group membership and other factors: misclassification can lead to attenuated or spurious associations with other variables (MacCallum, Zhang, Preacher, \& Rucker, 2002). Second, traditional dual-factor studies start from the assumption that there are four key groups of interest (e.g. Suldo $\&$ Shaffer, 2008), which precludes the idea that symptom domain (internalising or externalising) could be important in distinguishing individuals, or that intermediary levels of difficulties or strengths may be pertinent. Indeed, it has recently been argued that a greater range of mental health and well-being subtypes exist (Dowdy et al., 2015; Kim et al., 2017), suggesting that a less deductive approach is needed.

\section{Applying Latent Class Analysis to Investigate the Dual-Factor Model}

An alternative, inductive method for identifying mental health subtypes is latent class analysis (LCA). LCA is a datadriven statistical method which can be used to categorise individuals with similar patterns of behaviour (referred to as latent classes; Collins \& Lanza, 2010). Classes are derived so that each is internally homogenous and externally heterogeneous, overcoming some of the aforementioned difficulties with differentiation and misclassification. ${ }^{1}$ To date, those researching child mental health have typically used LCA to analyse patterns of symptoms, rather than taking a more comprehensive view that incorporates subjective well-being (Petersen, Qualter, \& Humphrey, 2019). Yet, in the adolescent mental health literature, a small number of studies have done so, yielding important findings. For example, Rose, Lindsey, Xiao, Finigan-Carr, and Joe (2017) identified four classes analogous to those found in traditional dual-factor studies in youth aged 13-17 years, and Moore, Dowdy, Nylund-Gibson, and Furlong (2019a, b) identified 'complete mental health', 'moderately mentally healthy', 'symptomatic but content', and 'troubled' classes, in adolescents in United States (US) grades 9-12, suggesting that common mental health types in adolescents align with categories predicted by the dual-factor model of mental health.

\footnotetext{
1 There are several benefits of applying LCA to study the dual-factor model in childhood. First, because mental health classes are not predetermined, this gives greater confidence that identified classes represent common patterns of mental health in the population rather than groups that have been 'forced' by applying cut-off points. However, given that LCA is a data-driven approach, steps should be taken to ensure that classes are not simply statistical artefacts (Bauer \& Curran, 2003). Where possible, it is important to check that classes are reliable, by testing that they are replicated in different samples, and valid, in that they co-vary with other variables as expected (Petersen, Qualter \& Humphrey, 2019). Second, using an inductive method allows the investigation of non-traditional groups. Dual-factor studies have generally focussed on four mental health groups identified by combining high/low mental symptoms and high/low subjective well-being. By using exploratory LCA, any number of classes, with any pattern of responses, across a range or distress and subjective well-being items are possible, meaning that other important groups may be identified. Finally, as classification error is accounted for in the model, there is greater confidence in any relations found between mental health classes and covariates, such as school connectedness and peer social support. Understanding common mental health subtypes and their covariates among schoolchildren has implications for how schools screen mental health, how they target interventions, and which factors they focus on in order to improve mental health.
} 


\section{The Dual-Factor Model in Middle Childhood}

To date, dual-factor studies that use latent class analysis have only investigated adolescent and adult mental health. In addition, traditional dual-factor studies have tended to focus on adolescent or adult populations (e.g. Antaramian et al., 2010; Lyons et al., 2012). There are a few exceptions, with evidence for the dual-factor model in children in school grades 3-6 in Canada ( $\mathrm{M}$ age $=10.5$ years, $\mathrm{SD}=0.7$; Greenspoon \& Saklofske, 2001) and with children in early elementary school (US kindergarten to $2^{\text {nd }}$ grade; Compton, 2016). More detailed investigation of the dual-factor model of mental health in middle childhood is necessary because mental health difficulties often emerge before adolescence, and elementary schools are increasingly seen as key institutions for promoting good mental health and preventing mental illness (Department of Health and Department for Education, 2017). The dual-factor model of mental health has important implications for addressing mental health in schools. By focussing on subjective well-being, as well as symptoms, children that may be vulnerable to mental health problems (i.e. low symptoms and low subjective well-being) can be identified in addition to those that are already symptomatic (Suldo \& Shaffer, 2008). Therefore, schools would be able to identify those in need of targeted mental health treatment and those that require a less intensive approach to prevent mental health problems developing. That would enable schools to allocate scarce resources efficiently and adopt proactive, as well as reactive, strategies for improving pupils' mental health. Therefore, research is needed to investigate whether mental health classes, similar to those predicted by the dual factor model of mental health, are found in middle childhood, how prevalent they are, and what factors influence membership of those mental health classes.

\section{The Present Study}

The present study aimed to address this gap in the literature by using LCA to investigate mental health classes in schoolchildren aged 8-9 years. A range of emotional difficulties, conduct problems, and subjective well-being items were included as mental health indicators so that a variety of mental health classes were possible, including those differentiated by type and level of distress or well-being.

In addition, the current study investigated whether the mental health classes were reliably found in different datasets and across genders. Reliability was explored by determining whether the same classes were replicated in different subsamples. Classes were expected to replicate in different datasets of similar children; however, the extent to which mental health classes would be replicated across genders could not be predicted based on previous literature. Studies that previously explored structural differences in mental health classes for males and females only investigated mental health symptoms and found mixed results, with some suggesting that classes were different (Althoff et al., 2006; Vendlinski et al., 2014), and others suggesting that they were equivalent (Baillargeon et al., 1999; Hudziak, Wadsworth, Heath, \& Achenbach, 1999; Wadsworth, Hudziak, Heath, \& Achenbach, 2001). Structural differences can affect the interpretation of results (Collins \& Lanza, 2010), so we investigated whether males and females had structurally different mental health classes, prior to investigating whether the prevalence of males and females in each class differed.

We also investigated the external validity of identified mental health classes by exploring whether they had distinct and expected relations with other factors. Family poverty, neighbourhood disadvantage, and special educational needs and disabilities (SEND) status have been linked to poor mental health and well-being (e.g. Pryor, Strandberg-Larsen, Nybo Andersen, Hulvej Rod, \& Melchior, 2019; Vedi \& Bernard, 2012). Therefore, we predicted that there would be stronger relations between those factors and classes indicating poor mental health. Conversely, high levels of school connectedness and social support are associated with lower levels of mental health symptoms and higher well-being (Monahan, Oesterle, \& Hawkins, 2010; Patalay \& Fitzsimons, 2016), so we predicted those variables would be associated with classes indicating good mental health. Furthermore, research suggests that prior academic attainment predicts later mental health (Deighton et al., 2018; Panayiotou \& Humphrey, 2018), and so positive relations were predicted between prior academic attainment and membership of good mental health classes.

\section{Method}

\section{Participants and Procedure}

This study was a secondary analysis of data collected for two randomised control trials (RCTs) of universal school-based interventions in the UK. Further information on dataset 1 (D1) can be found in Humphrey et al. (2018a) and further information on dataset 2 (D2) can be found in Humphrey et al. (2018b). Only data from children in the control arm of each trial were used because the interventions' logic models predicted changes in mental health. In addition, only data collected from children aged 8-9 years were analysed so that participants were comparable in age. Sample characteristics are presented in Table 1. 
Table 1 Sample characteristics

\begin{tabular}{|c|c|c|}
\hline Variable & Number $(\%) /$ Mean (SD) & \\
\hline$N$ & 3340 & \\
\hline Number of schools & 61 & \\
\hline Gender & Males $=1788(53.5 \%)$ & \\
\hline SEND & $631(19.3 \%)$ & \\
\hline FSM eligibility & $801(24.5 \%)$ & \\
\hline \multicolumn{3}{|l|}{ Ethnicity* } \\
\hline White & $2220(68.2 \%)$ & \\
\hline Black & $373(11.5 \%)$ & \\
\hline Asian & $371(11.4 \%)$ & \\
\hline Chinese & $14(0.4 \%)$ & \\
\hline Mixed ethnicity & $197(6.1 \%)$ & \\
\hline Other ethnicity & $70(2.2 \%)$ & \\
\hline Unclassified & $8(0.2 \%)$ & \\
\hline Mean social support score & $16.71(3.54)$ & \\
\hline Mean school connectedness score & $16.91(3.02)$ & \\
\hline IDACI score & $0.31(0.20)$ & \\
\hline KS1 Maths points average & $15.75(3.64)$ & \\
\hline KS1 English points average & $15.15(3.86)$ & \\
\hline SDQ conduct problems subscale & $0.89(1.63)$ & \\
\hline \multirow[t]{2}{*}{ SDQ emotional symptoms subscale } & $1.31(2.00)$ & \\
\hline & Overall endorsement & By category: somewhat/certainly \\
\hline \multicolumn{3}{|l|}{ Conduct problem item endorsement } \\
\hline Temper tantrums... & $464(15.3 \%)$ & $331(10.9 \%) / 133(4.4 \%)$ \\
\hline Generally obedient (R) & $805(26.5 \%)$ & $675(22.3 \%) / 130(4.3 \%)$ \\
\hline Often fights & $453(14.9 \%)$ & $362(11.9 \%) / 91(3.0 \%)$ \\
\hline Often lies or cheats & $408(13.5 \%)$ & $316(10.4 \%) / 92(3.0 \%)$ \\
\hline \multicolumn{3}{|l|}{ Emotional symptom item endorsement } \\
\hline Head-aches etc. & $594(19.6 \%)$ & $436(14.4 \%) / 158(5.2 \%)$ \\
\hline Many worries... & $884(29.2 \%)$ & $728(24.0 \%) / 156(5.1 \%)$ \\
\hline Unhappy, downhearted... & $579(19.1 \%)$ & $486(16.0 \%) / 93(3.1 \%)$ \\
\hline Nervous, or clingy... & $728(24.1 \%)$ & $607(20.1 \%) / 121(4.0 \%)$ \\
\hline Many fears... & $558(18.4 \%)$ & $459(15.1 \%) / 99(3.3 \%)$ \\
\hline \multicolumn{3}{|l|}{ Subjective well-being items mean } \\
\hline Enjoyable & $4.08(1.04)$ & \\
\hline Good mood & $3.90(1.01)$ & \\
\hline Had fun & $4.24(0.99)$ & \\
\hline Happy with way you are & $4.24(1.04)$ & \\
\hline
\end{tabular}

FSM = free school meals; IDACI $=$ income deprivation affecting children index; $\mathrm{R}=$ reverse scored; SEND $=$ special educational needs and disabilities

*Ethnic categories are those specified in the National Pupil Database. Note that 'Chinese' is a stand-alone group rather than a subset of the ‘Asian' category

\section{Measures}

\section{Mental Health Indicators}

Mental health difficulties were assessed using individual items from the emotional symptoms and conduct problems subscales of the English (UK) version of Strengths and Difficulties Questionnaire (SDQ) teacher-report version (Goodman, 1997). The overall scale has been found to have strong psychometric properties with 4-12 year olds (Stone, Otten, Engels, Vermulst, \& Janssens, 2010). The conduct problems items were (1) Often has temper tantrums or hot tempers, (2) Generally obedient, usually does what adults request (reverse scored), (3) Often fights with other children 
or bullies them, and (4) Often lies or cheats. ${ }^{2}$ The emotional symptoms items were (1) Often complains of headaches, stomach-aches or sickness, (2) Many worries, often seems worried, (3) Often unhappy, down-hearted or tearful, (4) Nervous or clingy in new situations, easily loses confidence and 5) Many fears, easily scared.

The SDQ asks teachers to rate whether the statements are 'not true', 'somewhat true', or 'certainly true'. For the LCA, responses were dichotomised so that $0=$ 'not true' and $1=$ 'somewhat' or 'certainly true'. Although some information about symptom severity was lost, dichotomisation reduced model complexity, which was necessary for the model to be able to run and converge on a final solution in Mplus. Similar studies have followed this procedure (e.g. Wadsworth et al., 2001).

Subjective well-being was measured using the positively worded items from psychological well-being scale of the self-report version of the KIDSCREEN-27, which has been validated with this age group (Ravens-Sieberer et al., 2007). ${ }^{3}$ The items included in the LCA were 'In the last week...' (1) Has your life been enjoyable? (2) Have you been in a good mood?, (3) Have you had fun?, and (4) Have you been happy with the way you are?. Children responded on a 5-point Likert scale from, $1=$ 'not at all' or 'never', to $5=$ 'extremely' or 'always. Treating responses as categorical resulted in a complex model, leading to model estimation difficulties. Unlike the SDQ items, the distribution of responses did not indicate a clear point for dichotomisation and there was no precedent for dichotomising those scores. Further, the items were rated on a 5-point Likert scale, which, arguably, can be treated as continuous data (Rhemtulla, Brosseau-Liard \& Savalei, 2012). Therefore, those items were treated as continuous.

\section{Covariates}

Several covariates were included as predictors of latent class membership.

Sociodemographic information was obtained through the UK National Pupil Database, (see, Jay, Mc Grath-Lone, \& Gilbert, 2019). Sociodemographic data were: gender, eligibility for free school meals (FSM), SEND status, and Income Deprivation Affecting Children Index (IDACI) score. The IDACI measured relative levels of deprivation in small areas of England called Lower-layer Super Output Areas (LSOAs). Scores range from 0 to 1 which can be

\footnotetext{
${ }^{2}$ Sensitivity analysis indicated that a fifth conduct problems SDQ item 'steals from home school or elsewhere' was not a suitable indicator because it was rarely endorsed, therefore, this was not included in the LCA analysis. Other studies have also found this item to be problematic (Panayiotou \& Humphrey, 2018).

${ }^{3}$ Negatively worded items were not included in the analysis because they overlapped with items in the SDQ emotional symptoms subscale.
}

interpreted as the proportion of families in an LSOA with children aged under 16 years, who are income deprived.

Peer support and school connectedness were assessed using 'social and peer support' and 'school environment' self-report subscales from the KIDSCREEN-27 (RavensSieberer et al., 2007). Both subscales have four items and responses on a 5-point Likert scale. Total scores ranged from 4 to 20 , with high scores indicating greater perceived social support or school connectedness. Both subscales have good internal reliability (Cronbach's alpha for both scales $=0.81$; Robitail et al. 2007), criterion validity (correlations with scales from the KIDSCREEN-52 item questionnaire of $r=0.96$ ), and good test-retest reliability over a 2 -week period (ICC $=0.61$ and 0.74 , respectively; Ravens-Sieberer et al., 2007).

Prior academic attainment was measured through Key Stage 1 national assessment scores in Maths and English, obtained from the UK National Pupil Database. These are statutory teacher assessments of children aged 6-7 years. Scores are based on government issued assessment frameworks (Standards and Testing Agency, 2018). Scores range from 3 to 30 .

\section{Data Analysis}

Analyses were conducted in Mplus v8.2, using maximum likelihood estimation and an expectation-maximisation (EM) algorithm (Muthén \& Muthén, 1998-2017). The complex structure of the data, due to clustering by school, was accounted for using a sandwich estimator.

The number of mental health classes in the population was previously unknown so exploratory LCA with both binary and continuous indicators was conducted: we fit an unrestricted latent class model with 1-class, and then with an increasing number of classes, until convergence issues were encountered. To prevent convergence on local maxima, the analyses were run with 1000 start values with 200 optimisations and 50 iterations.

For each model, Akaike Information Criteria (AIC), Bayesian Information Criteria (BIC), and sample size adjusted Bayesian Information Criteria (ssaBIC) were recorded. Lower values on information criteria indicated better model fit (Hagenaars \& McCutcheon, 2002). The Lo-Mendell-Rubin Likelihood Ratio Test (LMR-LRT) statistic was also recorded. Significant LMR-LRT results indicated that the model with K-classes was a significant improvement over the model with K-1 classes (Masyn, 2013). Monte Carlo simulations have indicated that when a mixture of categorical and continuous variables is included in LCA, those fit indices perform well. ssaBIC and BIC generally performed best with large samples ( $>1200)$, although, when rare classes are identified, AIC performed better (Morgan, 2015). 
Classification accuracy for each model was recorded. This was not used for class enumeration purposes because it is not a fit statistic; instead, it provided information about the quality of classification provided by each model (Masyn, 2013). Entropy values and average latent class probabilities close to 1 indicated precise classification; values above 0.8 were considered good (Clark \& Muthén, 2009).

Fit statistics were used to reduce the total number of models to a smaller number of candidate models. Thereafter, a number of substantive criteria were considered. First, item response and associated plots were examined to assess whether classes were homogeneous, (i.e. whether members of the same class provided the similar responses). They were also examined to assess whether there was good class separation (i.e. each class was distinct from the others). Item response probabilities close to 0 or 1 indicated good class homogeneity, whereas good latent class separation was indicated if there was a unique and characteristic response pattern associated with each class (Collins \& Lanza, 2010). Second, the interpretability of classes was also assessed; specifically, whether we could interpret classes in line with theory and research. Third, models that identified classes containing less than $5 \%$ of the sample were disregarded because small classes can be unstable (Masyn, 2013) and would have too few members to enable later latent class regression analyses. Parsimony was a final consideration.

Missing data on the LCA indicators were accounted for by Full Information Maximum Likelihood (FIML; Enders, 2010).

\section{Replicating the Latent Classes Across Datasets and Genders}

Initially, the overall dataset was split into four subsamples, by gender and dataset: D1 males $(n=727)$, D1 females $(n=604)$, D2 males $(n=1061)$ and D2 females $(n=948){ }^{4}$ Separate latent class analyses were conducted for each subgroup to determine whether the same number and type of classes were identified. Multiple-group LCA (Collins \& Lanza, 2010) was used to test whether the classes were structurally equivalent in each subgroup. BIC, AIC and Chi square difference test results were recorded for the following models: (1) an unrestricted model, (2) a model assuming structural equivalence, and (3) a model assuming structural equivalence and equivalence of class prevalences. Smaller fit indices for the restricted model and non-significant Chi square difference tests indicated model equivalence.

\footnotetext{
${ }^{4}$ Characteristics of each sample are presented online (Online Resource 1).
}

\section{Combining Datasets}

Combining data from the subsamples of data confers the advantage of having a larger sample size, which increases statistical power. That is particularly advantageous in the final step of the analysis, which incorporates covariates into the model. Participants in each subsample were from the same age group and from a similar geographic area, indicating suitability for combining data. However, because subsamples had some different characteristics (see online material 1), we only considered it appropriate to combine data from the different subsamples when the structural equivalence of mental health classes was indicated. If structural equivalence of classes could not be assumed, data would not be combined and separate regression analyses would be carried out for each subgroup. However, if structural equivalence of classes could be assumed across subsamples (either gender, dataset, or both), data would be combined to increase the sample size for the latent class regression analysis. In this case, the class enumeration process was repeated with the combined dataset, confirming the number of classes in the best fitting model, since sample size can influence this process (Nylund, Asparouhov, \& Muthén, 2007).

\section{Adding Predictor Variables}

Predictor variables were incorporated into the final latent class model using the 3-step approach (Asparouhov \& Muthén, 2014), which preserved the classes identified in the class enumeration stage of the analysis, while accounting for measurement error. The predictors were then regressed onto the classes. The 3-step method is the most appropriate for examining the relations between classes and covariates (Asparouhov \& Muthén, 2018). Cases with missing data on covariates were deleted list-wise by default in Mplus.

\section{Results}

\section{Multiple-Group Latent Class Analyses}

Separate analyses for each subsample, based on gender and dataset, indicated a 3- or 4-class model best fit the data (see Online Resource 2). Examination of responses indicated that both the 3-and 4-class models were structurally comparable across subsamples (Online Resource 3). Further, it was our opinion that any difference in responses between subgroups did not alter the substantive meaning of the class.

In the multiple-group analysis, BIC indicated that restricted models showed better model fit than the unrestricted model, suggesting classes were structurally equivalent across samples. Conversely, AIC indicated that the unrestricted model fit the data best. The discrepancy likely 
Table 2 Fit statistics, classification indices, and class sizes for each model

\begin{tabular}{lllllllll}
\hline K & LL & AIC & BIC & ssaBIC & LMR-LRT $p$ & $\begin{array}{l}\text { Entropy } \\
\text { Average class } \\
\text { probabil- } \\
\text { ity }>0.8\end{array}$ & Class size (proportion based on model estimate) \\
& & & & & & & & \\
\hline 1 & -31308.52 & 62651.05 & 62754.98 & 62700.97 & $\mathrm{n} / \mathrm{a}$ & $\mathrm{n} / \mathrm{a}$ & $\mathrm{n} / \mathrm{a}$ & 1.0 \\
2 & -29605.31 & 59272.62 & 59462.14 & 59363.64 & 0.0124 & 0.74 & $\mathrm{y}$ & $0.36 / 0.64$ \\
3 & -28377.62 & 56845.23 & 57120.35 & 56977.36 & 0.0026 & 0.80 & $\mathrm{y}$ & $0.16 / 0.24 / 0.60$ \\
$\mathbf{4}$ & $-\mathbf{2 7 7 4 3 . 3 9}$ & $\mathbf{5 5 6 0 4 . 7 7}$ & $\mathbf{5 5 9 6 5 . 4 8}$ & $\mathbf{5 5 7 7 8 . 0 1}$ & $\mathbf{0 . 0 2 2 9}$ & $\mathbf{0 . 8 2}$ & $\mathbf{y}$ & $\mathbf{0 . 1 2 / 0 . 1 3 / 0 . 1 8 / 0 . 5 7}$ \\
5 & -27391.97 & 54929.94 & 55376.24 & 55144.29 & 0.1076 & 0.78 & $\mathrm{y}$ & $0.07 / 0.12 / 0.18 / 0.20 / 0.44$ \\
6 & -27174.00 & 54522.00 & 55053.89 & 54777.46 & 0.6432 & 0.80 & $\mathrm{y}$ & $0.04 / 0.05 / 0.11 / 0.16 / 0.20 / 0.43$ \\
7 & -26935.82 & 54073.63 & 54691.12 & 54370.20 & 0.3056 & 0.81 & $\mathrm{y}$ & $0.04 / 0.05 / 0.07 / 0.10 / 0.15 / 0.16 / 0.43$ \\
8 & -26765.95 & 53761.90 & 54464.98 & 54099.57 & 0.3304 & 0.81 & $\mathrm{n}$ & $0.04 / 0.04 / 0.05 / 0.07 / 0.10 / 0.14 / 0.16 / 0.40$ \\
9 & -26608.81 & 53475.63 & 54264.30 & 53854.40 & 0.4627 & 0.82 & $\mathrm{n}$ & $0.03 / 0.04 / 0.04 / 0.05 / 0.07 / 0.09 / 0.12 / 0.15 / 0.41$ \\
10 & -26471.27 & 53228.54 & 54102.80 & 53648.42 & 0.5434 & 0.82 & $\mathrm{n}$ & $0.03 / 0.03 / 0.04 / 0.04 / 0.05 / 0.07 / 0.10 / 0.11 / 0.14 / 0.40$ \\
\hline
\end{tabular}

$\mathrm{AIC}=$ Akaike Information Criterion; BIC $=$ Bayesian Information Criterion; $\mathrm{K}=$ number of classes; LL $=$ log-likelihood; LMR-LRT $=$ LoMendell-Rubin Likelihood Ratio Test; $\mathrm{p}=p$ value; $\mathrm{ssaBIC}=$ sample size adjusted BIC

Bold $=$ final 4-class solution

reflects that AIC does not penalise model complexity as heavily as BIC. The Chi square difference test indicated that class structures were different across subgroups (Online Resource 4). Despite not passing the very strictest tests of class equivalence between groups, we considered there to be enough evidence to assume equivalence of the 3- and 4-class models across groups. As such, the data were pooled for the next stage of analysis.

\section{Final Latent Class Model Using Combined Data}

The LCA class enumeration process was repeated for the final combined dataset. The information criteria and LMRLRT indicated that the 4-class model best fit the data. Entropy was good (0.82) and classification for each class was accurate (average class probabilities for most likely class were all over 0.8 ; see Table 2 and Fig. 1). In addition, class separation was good: each mental health class had clearly distinguishable characteristics (see Fig. 2). Each class was interpretable in line with existing mental health frameworks, specifically the dual-factor model (Greenspoon \& Saklofske, 2001) and dimensional models of internalising and externalising symptoms (Achenbach \& Edelbrock, 1978). No class contained less than $5 \%$ of the sample. The final four classes were as follows: (1) complete mental health $(n=1895,57 \%)$, characterised by very low probability of symptom endorsement and high subjective well-being scores; (2) vulnerable $(n=434,13 \%)$, characterised by slightly elevated probability of endorsing mental health symptoms and below average subjective well-being; (3) conduct problems but content $(n=404,12 \%)$, characterised by high probability of endorsing conduct problems items, moderate probability of endorsing emotional symptoms, but high subjective well-being; and (4) emotional symptoms but content $(n=606,18 \%)$, characterised by low probability of endorsing conduct problems, high probability of endorsing emotional symptoms, and high subjective well-being (see Table 3).

\section{Testing the External Validity of Classes in the Final Model}

The LCA covariate analysis tested the external validity of the final class solution by investigating the relations between classes and other factors that influence mental health. 351 (10.5\%) cases with missing data on one or more of the covariates were deleted list-wise, leaving 2,989 children in the latent class regression analysis. Results showed that classes were distinct in their relation with predictor variables (see Table 4).

Compared to the complete mental health class, those in the emotional symptoms but content class were significantly more likely to be female, to have SEND, to live in more affluent neighbourhoods (indicated by lower IDACI scores), and to have lower prior attainment (maths scores). Those in the conduct problems but content class were significantly more likely to be male, have SEND, and report lower levels of school connectedness. Those in the vulnerable class were significantly more likely to experience familial poverty (indicated by higher uptake of FSM) and report lower levels of both social support and school connectedness.

Comparison of the two symptomatic but content groups indicated that those with high conduct problems were significantly more likely to be male and have lower levels of school connectedness. The vulnerable group differs from both symptomatic but content groups: they reported lower levels of social support and school connectedness, and were less likely to have SEND than those in the conduct problems but content group. 
Fig. 1 Akaike Information Criteria (AIC), Bayesian Information Criteria (BIC) and sample size adjusted Bayesian Information Criteria (ssaBIC) values for each class model. Lower values indicate better model fit

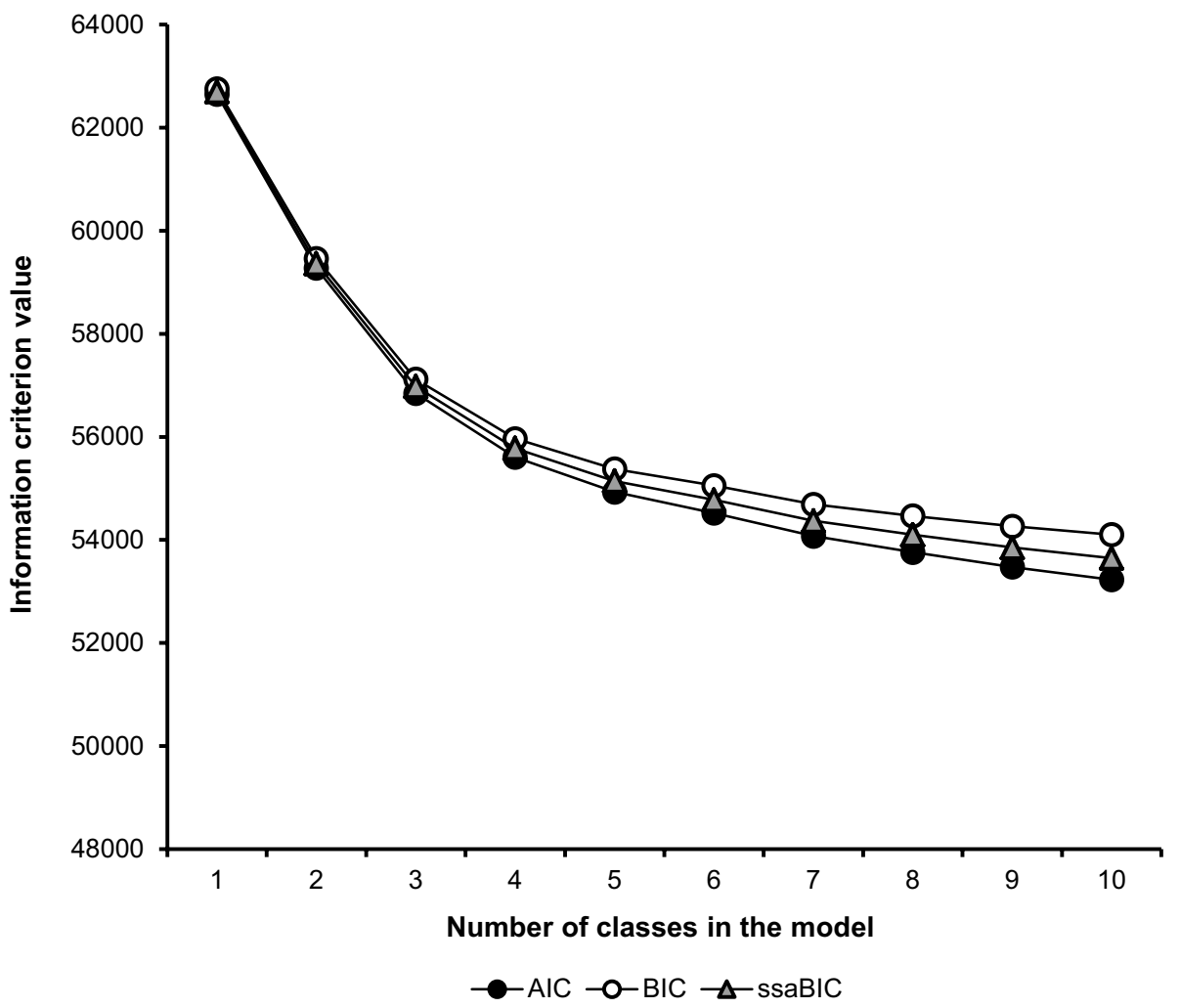

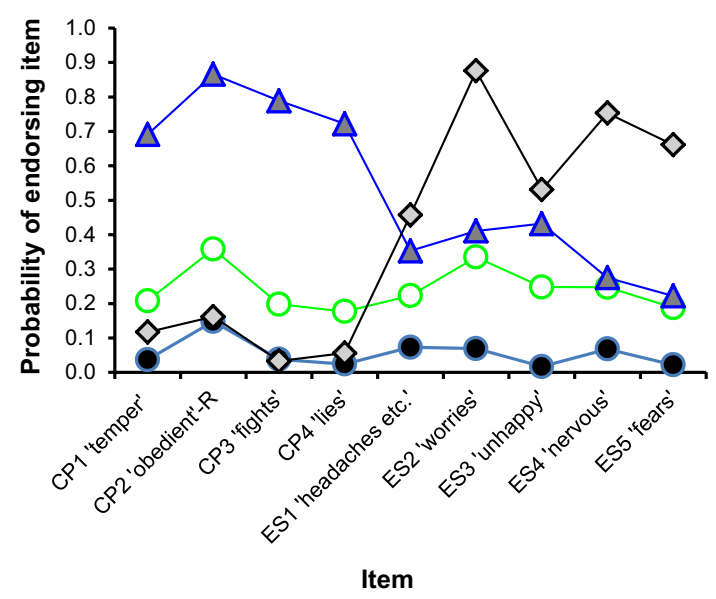

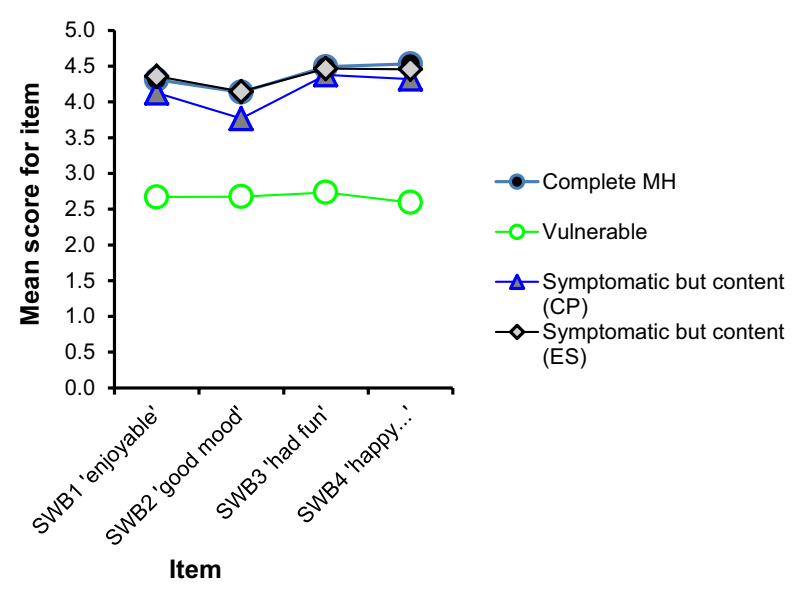

Fig. 2 Plot of a item response probabilities for endorsing conduct problems and emotional symptoms, and $\mathbf{b}$ mean scores for subjective wellbeing items, for each class

\section{Discussion}

This study used LCA to identify common patterns of mental health symptoms and subjective well-being in schoolchildren aged $8-9$ years. The analysis indicated that children could be categorised into four common classes, defined by a unique pattern of symptoms and subjective well-being. The identified latent classes were (1) complete mental health (57\% of sample), (2) vulnerable (13\%), (3) conduct problems but content (12\%), and (4) emotional symptoms but content (18\%). Those findings provide support for the dual-factor model of mental health, suggesting that symptoms of distress and subjective well-being are distinct constructs rather than opposite ends of a single mental health continuum (Greenspoon \& Saklofske, 2001). Children in the vulnerable class, and both symptomatic but content classes, showed patterns of symptoms 
Table 3 Final 4-class model, average responses per class

\begin{tabular}{|c|c|c|c|c|c|}
\hline Aspect of mental health & Item & $\begin{array}{l}\text { Complete mental } \\
\text { health }\end{array}$ & Vulnerable & $\begin{array}{l}\text { Emotional symptoms } \\
\text { but content }\end{array}$ & $\begin{array}{l}\text { Conduct } \\
\text { problems but } \\
\text { content }\end{array}$ \\
\hline \multirow[t]{4}{*}{ Conduct problems } & Temper tantrums... & 0.037 & 0.208 & 0.117 & 0.691 \\
\hline & Generally obedient... (R) & 0.148 & 0.358 & 0.162 & 0.866 \\
\hline & Often fights... & 0.038 & 0.198 & 0.033 & 0.789 \\
\hline & Often lies or cheats & 0.024 & 0.177 & 0.056 & 0.722 \\
\hline \multirow[t]{5}{*}{ Emotional symptoms } & Headaches... & 0.073 & 0.223 & 0.457 & 0.353 \\
\hline & Many worries... & 0.069 & 0.335 & 0.876 & 0.411 \\
\hline & Unhappy... & 0.017 & 0.248 & 0.531 & 0.432 \\
\hline & Nervous or clingy... & 0.068 & 0.247 & 0.753 & 0.275 \\
\hline & Many fears... & 0.022 & 0.188 & 0.661 & 0.222 \\
\hline \multirow[t]{4}{*}{ Subjective well-being } & Enjoyable & 4.312 & 2.670 & 4.359 & 4.127 \\
\hline & Good mood & 4.135 & 2.675 & 4.145 & 3.767 \\
\hline & Had fun & 4.492 & 2.734 & 4.467 & 4.380 \\
\hline & Happy with way you are & 4.533 & 2.594 & 4.457 & 4.321 \\
\hline
\end{tabular}

$\mathrm{R}=$ reverse scored

Conduct problems and emotional symptom item responses represent the average probability of the item being endorsed. Subject well-being scores are means

Item responses above 0.5 and means over 4 are in bold to aid interpretation of the table

and subjective well-being that would not be possible on such a continuum. The classes were reliably found across datasets and gender. In addition, latent class regression analysis indicated that classes were distinct and externally valid, as evidenced by their differential associations with sociodemographic and other factors.

It is notable that LCA, a data-driven, inductive approach, produced three of the classes logically indicated by the dual-factor model: complete mental health, vulnerable, and symptomatic but content (Antaramian et al., 2010; Suldo et al., 2011, 2016; Thalji, 2012). Further, the large complete mental health class and smaller vulnerable class were comparable in size to that found in other dual-factor studies (e.g. Antaramian et al., 2010; Compton, 2016; Kelly, Hills, Huebner, \& McQuillin, 2012; Rose et al., 2017; Suldo \& Shaffer, 2008; Suldo et al., 2011; Thalji, 2012).

A key difference between our findings and that of other dual-factor studies is that we identified two symptomatic but content groups rather than one, distinguished by the symptom domain. Further, the classes were found to have different sociodemographic and psychosocial predictors (i.e. gender and school connectedness), which attests to their distinctiveness and, perhaps, different aetiologies. Other research has suggested that children experience qualitative differences in mental health symptoms (Basten et al., 2013, 2016; McElroy, Shevlin, \& Murphy, 2017), yet many traditional dual-factor studies have not differentiated symptoms (e.g. Suldo \& Shaffer, 2008). Two studies that investigated internalising and externalising symptoms separately, along with levels of well-being (co-vitality) in adolescents (Kim et al., 2017; Rebelez Ernst, 2016), found that groups showed distinct relations with covariates, supporting our findings that symptom domain is an important component of mental health types.

Recent latent class, dual-factor research with adolescents identified a single symptomatic but content class, as opposed to the two identified presently (Moore et al., 2019a, b; Rose et al., 2017). Other LCA studies may not have identified two 'symptomatic but content classes' because they had smaller sample sizes (ranging from c.291-1170 participants), which made it difficult to identify small classes. Indeed, in our initial subsample analyses, information criteria generally favoured the three-class model, which only had one symptomatic but content group. When the larger combined dataset was used, the four-class model with two symptomatic but content classes was clearly indicated. Further research with large samples is therefore needed to investigate whether separate internalising and externalising symptomatic but content classes are replicated in older age groups.

The final subgroup predicted by the dual-factor model is the 'troubled' group, characterised by high symptoms and low subjective well-being. Previous studies have identified this group in adolescence (e.g. Antaramian et al., 2010; Moore et al., 2019a, b; Suldo \& Shaffer, 2008) and childhood (Compton, 2016; Greenspoon \& Saklofske, 2001). However, such a group was not identified through our analysis. Developmental research indicates that subjective well-being tends to remain high during 
Table 4 Odds ratios and 95\% confidence intervals showing the relationship between class and predictor variables

\begin{tabular}{|c|c|c|c|c|}
\hline & $\begin{array}{l}\text { Emotional symptoms but } \\
\text { content }\end{array}$ & Conduct problems but content & Vulnerable & $\begin{array}{l}\text { Complete } \\
\text { mental } \\
\text { health }\end{array}$ \\
\hline \multicolumn{5}{|c|}{ Complete mental health class as reference class } \\
\hline Male & $0.641(0.485-0.846)$ & $1.472(1.049-2.066)$ & $0.952(0.661-1.370)$ & - \\
\hline Dataset 1 & $0.804(0.512-1.260)$ & $0.747(0.390-1.432)$ & $0.801(0.516-1.244)$ & - \\
\hline FSM & $1.085(0.784-1.501)$ & $1.507(0.996-2.281)$ & $1.581(1.064-2.350)$ & - \\
\hline With SEND & $2.005(1.342-2.997)$ & $2.267(1.514-3.394)$ & $1.201(0.685-2.106)$ & - \\
\hline IDACI score & $0.303(0.093-0.989)$ & $0.755(0.199-2.868)$ & $0.625(0.233-1.678)$ & - \\
\hline English & $1.011(0.952-1.074)$ & $0.964(0.896-1.038)$ & $0.989(0.920-1.064)$ & - \\
\hline Maths & $0.918(0.856-0.984)$ & $0.927(0.858-1.002)$ & $0.976(0.895-1.064)$ & - \\
\hline Social support & $0.965(0.921-1.011)$ & $1.024(0.958-1.094)$ & $0.804(0.765-0.845)$ & - \\
\hline School connectedness & $0.998(0.936-1.064)$ & $0.747(0.704-0.792)$ & $0.658(0.614-0.705)$ & - \\
\hline \multicolumn{5}{|c|}{ Emotional symptoms but content as reference class } \\
\hline Male & - & $2.298(1.468-3.596)$ & $1.486(0.942-2.345)$ & - \\
\hline Dataset 1 & - & $0.929(0.578-1.494)$ & $0.997(0.586-1.695)$ & - \\
\hline FSM & - & $1.389(0.857-2.252)$ & $1.458(0.916-2.319)$ & - \\
\hline With SEND & - & $1.130(0.671-1.906)$ & $0.599(0.298-1.205)$ & - \\
\hline IDACI score & - & $2.493(0.685-9.078)$ & $2.063(0.465-9.147)$ & - \\
\hline English & - & $0.954(0.893-1.018)$ & $0.979(0.896-1.069)$ & - \\
\hline Maths & - & $1.010(0.931-1.097)$ & $1.064(0.963-1.174)$ & - \\
\hline Social support & - & $1.061(0.985-1.142)$ & $0.833(0.791-0.878)$ & - \\
\hline School connectedness & - & $0.749(0.693-0.809)$ & $0.659(0.608-0.715)$ & - \\
\hline \multicolumn{5}{|c|}{ Conduct problems but content as reference class } \\
\hline Male & - & - & $0.647(0.414-1.010)$ & - \\
\hline Dataset 1 & - & - & $1.073(0.589-1.954)$ & - \\
\hline FSM & - & - & $1.049(0.662-1.662)$ & - \\
\hline With SEND & - & - & $0.530(0.300-0.935)$ & - \\
\hline IDACI score & - & - & $0.828(0.192-3.573)$ & - \\
\hline English & - & - & $1.026(0.938-1.123)$ & - \\
\hline Maths & - & - & $1.053(0.958-1.157)$ & - \\
\hline Social support & - & - & $0.785(0.734-0.840)$ & - \\
\hline School connectedness & - & - & $0.880(0.822-0.944)$ & - \\
\hline
\end{tabular}

IDACI = income deprivation affecting children index; FSM = eligible for free school meals; SEND = special educational needs and disabilities Bold $=$ Significant relationships, i.e. $95 \%$ confidence intervals do not cross 1

the elementary school years and then declines around 10 years old (Casas \& Gonzalez-Carrasco, 2019). Further, emotional symptoms and conduct problems are relatively uncommon in childhood and increase with age (Polanczyk, Salum, Sugaya, Caye, \& Rohde, 2015). Owing to the low base-rate of children with low subjective well-being, in addition to a low base-rate of children with mental health difficulties, the numbers of children aged 8-9 years with simultaneously high symptoms and low subjective wellbeing is likely to be low. In our analyses we rejected latent class models which had classes that represented less than $5 \%$ of the sample because such groups can be unstable (Masyn, 2013). Therefore, the lack of a troubled group may be attributable to the age of the sample and statistical decisions made during the model selection process.

A strength of our study is that we replicated the LCA in four different subsamples. Mixture modelling approaches, like LCA, have been criticised for producing results that are sample specific (Bauer \& Curran, 2004). Exploratory LCA, in particular, may be susceptible to this because it is a datadriven approach. The final four-class model was most consistent across all four samples and was the best fitting model when the data were combined. However, further research is required to determine whether those results are replicated in other samples, including those in other countries.

Carrying out LCA for males and females separately, in the first instance, also allowed for detailed investigation 
of the mental health classes across genders. Classes were structurally analogous for males and females, which is consistent with research investigating classes of particular mental health symptoms, such as obsessions and compulsions (Althoff, Rettew, Boomsma, \& Hudziak, 2009), anxiety and depression (Wadsworth et al., 2001), and aggression (Baillargeon et al., 1999). Although classes were structurally similar, the proportion of males and females in each class differed significantly. In particular, females were significantly more likely to be in the 'emotional symptoms but content' class and males in the 'conduct problems but content' class. This finding is in line with research that indicates males are more likely to display conduct problems, whereas females are more likely to display emotional symptoms (Keiley, Bates, Dodge, \& Pettit, 2000).

Another strength of the study was the examination of class validity. External validity was indicated by classes showing expected relations with other factors. For example, mental health risk factors, such as SEND status, low prior Maths attainment and having low perceived peer support and school connectedness, were associated with less adaptive mental health classes. Associations were consistent with other research in this field (e.g. McLeod \& Kaiser, 2004; Monahan et al., 2010; Patalay \& Fitzsimons, 2016). The only unexpected result was the association between membership of the 'emotional symptoms but content' class and increased neighbourhood affluence. Neighbourhood poverty is generally considered a risk factor for mental health (Boyle et al., 2019). Because this class was associated with being female, having lower prior academic attainment, and being in a more affluent area, it is possible that symptom expression is rooted in a sense of failing to live up to the high expectations of parents and teachers. Future research should explore that further.

Beyond those general expected associations, the study findings provided a more nuanced account of how sociodemographic and psychosocial factors relate to more complex patterns of mental health. For example, family poverty, low social support, and low school connectedness were more strongly associated with membership of the vulnerable class than any other class. Those children have lower than average well-being and burgeoning symptoms, and research suggests they may be at risk of developing more severe symptoms later in life (Wang, Zhang, \& Wang, 2011). Although our cross-sectional study cannot be used to infer causality, findings suggest that addressing the negative effects of poverty and helping youth to develop good peer relationships and school connectedness may benefit this vulnerable group. However, further research is necessary to ascertain the reliability of our initial findings.

\section{Limitations}

There are several limitations of the current study. First, mental health and psychosocial data were collected concurrently, so causal direction cannot be inferred. In addition, we do not know whether the classes have predictive validity and longitudinal research is needed.

Second, mental health symptoms were teacher-reported. This is in line with previous studies that investigated the dual factor model of mental health in children (i.e. Compton, 2016; Greenspoon \& Saklofske, 2001) and reflects the fact that many measures of child mental health are not available for self-report in this age group. However, while teachers may be good reporters of conduct problems, they are less qualified to report on children's internal states (De Los Reyes, 2015; Papandrea \& Winefield, 2011). To test the reliability of our findings when children self-report symptoms, future research should consider an alternative, age-appropriate self-report measure, such as the Me and My School questionnaire (Deighton et al., 2013).

Third, we identified peer support and school connectedness as correlates with class membership. Future research will need to examine other forms of social support, different components of school connectedness, or mediator and moderator effects, to explore those relations more fully.

Fourth, we acknowledge that our assessment of subjective well-being was somewhat limited. Typically, subjective well-being is defined as overall positive affect and life satisfaction (Lucas, 2016). A separate measure of life satisfaction was not available in the datasets used for our analysis. Measurement shortcomings are a limitation of many studies that use secondary data because the specific research questions were not the immediate focus of the research team collecting the data. It is possible that incorporating a more extensive measure of life satisfaction into the analysis would lead to the identification of different mental health classes. Where possible, future research should incorporate a measure of life satisfaction to investigate whether results are altered. A related consideration is that subjective well-being can be argued to include more than the appraisal of affect and life satisfaction, encompassing other aspects, such as social well-being. Such a broad definition of subjective well-being was not the focus of the current study and discussion of such conceptual discrepancies are beyond the scope of this article (see Lima \& Morais, 2018; Lucas, 2016, for further discussion on this issue). However, we note that encompassing more aspects of well-being into a similar latent class model may lead to the identification of mental health and well-being classes that are less focussed on symptomology, which may be of substantive interest.

A further limitation regards treatment of items used in the latent class analysis. In order to simplify the latent 
class model so that it would converge on a final solution, SDQ symptom responses, which were originally on a 3-point scale, were dichotomised and KIDSCREEN wellbeing items, which were on a 5-point scale, were treated as continuous. Therefore, some information from the original scale was lost. Further, KIDSCREEN items were negatively skewed, with high ceiling effects, which can be problematic when treating ordinal variables as continuous (Bauer \& Curran, 2003). Further research will need to test the reliability of these classes with measures that are less prone to skew or using ordinal variables where the analysis allows.

Finally, the generalisability of the results is subject to certain limitations: participants were recruited from schools that volunteered to take part in RCTs of universal interventions and may not be representative of children in the wider UK population. In addition, the sample could be further biased through the handling of missing data. Missing data were handled using the default settings in Mplus because there is no strong consensus on a more effective way to handle missing data on covariates (Heron, Croudace, Barker, \& Tilling, 2015). Cases with missing data on predictor variables were deleted list-wise; the remaining sample size was still very large $(n=2989)$ and well powered, although it is possible list-wise deletion introduced bias.

\section{Implications}

Our study demonstrated the importance of simultaneously measuring internalising and externalising symptoms and subjective well-being when investigating children's mental health. Most child mental health research is variable centred, examining the relation between a single aspect of mental health and other factors. However, it appears that patterns of mental health symptoms and subjective wellbeing define groups of children. Failing to consider those patterns of mental health could lead to unreliable findings, and the complex interaction between different symptoms and well-being would be lost. Therefore, we recommend that research investigating child mental health incorporates methods, such as latent class analysis, which can consider a range of aspects of mental health, in order to supplement more traditional variable centred approaches.

Similarly, when screening children's mental health in elementary schools, a full range of symptoms and indicators of subjective well-being should be considered. This would allow schools to identify both symptomatic and vulnerable children, so that interventions can be appropriately targeted. Different groups may require different approaches. For example, those with conduct problems and emotional symptoms may require specific interventions or referrals to other mental health services; those with low symptoms and low well-being may benefit from lower intensity, preventative interventions in school.

Further, elementary schools often implement universal intervention programs to improve the mental health and well-being of their pupils (Patalay et al., 2017). However, despite children receiving the same intervention, different outcomes might be expected for particular subgroups of children (Greenberg \& Abenavoli, 2017). For example, those with complete mental health may show less improvement because they are already showing good mental health; symptomatic individuals may only show slight improvement if they require more intensive interventions; and vulnerable individuals may show the greatest improvement over time if the intervention is preventative. Therefore, a suggestion for researchers and educators that evaluate the impact of universal mental health programmes is to investigate the effect of the intervention for each group. That would give a more detailed account of the effect of those interventions and prevent the loss of information which would occur through aggregating scores across the whole sample.

Finally, the findings demonstrate that subjective wellbeing is a key component of child mental health. This suggests the importance of interventions that focus on improving well-being as well as reducing symptoms. To date, a number of school-based positive psychology interventions have been developed that are associated with increased subjective well-being amongst children (e.g. Quinlan, Swain, Cameron, \& Vella-Brodrick, 2015; Suldo et al., 2015; Wingate, Suldo, \& Peterson, 2018). Those interventions focus on the building blocks of subjective well-being, such as developing peer and student-teacher relationships, as well as using character strengths-based interventions to evoke positive feelings. Our findings regarding the relational correlates of the identified mental health classes in the current study provide foundational support for such work.

Acknowledgements This research was supported by a grant from the National Institute of Health Research (Grant References 14/52/38 and 10/3006/01).

Author Contributions All authors contributed to the study conception and design. Data analysis was conducted by Kimberly J. Petersen. The first draft of the manuscript was written by Kimberly J. Petersen and all authors commented on previous versions of the manuscript. All authors read and approved the final manuscript.

\section{Compliance with Ethical Standards}

Conflict of interest The authors declare that they have no conflict of interest.

Ethical Approval This study was a secondary data analysis. The original studies were approved by the University Research Ethics Committee at the University of Manchester, UK. Informed 'opt-out' consent from the parents/carers of participants and participant assent were obtained for all participants in the original studies. 
Open Access This article is licensed under a Creative Commons Attribution 4.0 International License, which permits use, sharing, adaptation, distribution and reproduction in any medium or format, as long as you give appropriate credit to the original author(s) and the source, provide a link to the Creative Commons licence, and indicate if changes were made. The images or other third party material in this article are included in the article's Creative Commons licence, unless indicated otherwise in a credit line to the material. If material is not included in the article's Creative Commons licence and your intended use is not permitted by statutory regulation or exceeds the permitted use, you will need to obtain permission directly from the copyright holder. To view a copy of this licence, visit http://creativecommons.org/licenses/by/4.0/.

\section{References}

Achenbach, T. M., \& Edelbrock, C. S. (1978). The classification of child psychopathology: A review and analysis of empirical efforts. Psychological Bulletin, 85(6), 1275-1301.

Althoff, R. R., Copeland, W. E., Stanger, C., Derks, E. M., Todd, R. D., Neuman, R. J., et al. (2006). The latent class structure of ADHD is stable across informants. Twin Research and Human Genetics, 9(4), 507-522.

Althoff, R. R., Rettew, D. C., Boomsma, D. I., \& Hudziak, J. (2009). Latent class analysis of the child behavior checklist obsessivecompulsive scale. Comprehensive Psychiatry, 50(6), 584-592.

Antaramian, S. P., Huebner, E., Hills, K. J., \& Valois, R. F. (2010). A dual-factor model of mental health: Toward a more comprehensive understanding of youth functioning. American Journal of Orthopsychiatry, 80(4), 462-472.

Asparouhov, T., \& Muthén, B. (2014). Auxiliary variables in mixture modeling: Three-step approaches using Mplus. Structural Equation Modeling, 21(3), 329-341.

Asparouhov, T., \& Muthén, B. (2018). Auxiliary variables in mixture modeling: Using the $\mathrm{BCH}$ method in Mplus to estimate a distal outcome model and an arbitrary secondary model. Mplus Web Notes, 21(2), 1-22.

Baillargeon, R. H., Tremblay, R. E., \& Willms, D. (1999). The prevalence of physical aggression in Canadian children: A multi-group latent class analysis of data from the first collection cycle (19941995) of the NLSCY. Retreived September 17, 2019, from https ://ruor.uottawa.ca/bitstream/10393/12870/1/Baillargeon_Raymo nd_1999_The_prevalence_of_physical_aggression_in_Canad ian_children.pdf.

Basten, M. M., Althoff, R. R., Tiemeier, H., Jaddoe, V. W., Hofman, A., Hudziak, J. J., et al. (2013). The dysregulation profile in young children: empirically defined classes in the Generation R study. Journal of the American Academy of Child and Adolescent Psychiatry, 52(8), 841-850.

Basten, M. M., Tiemeier, H., Althoff, R. R., van de Schoot, R., Jaddoe, V. W., Hofman, A., et al. (2016). The stability of problem behavior across the preschool years: An empirical approach in the general population. Journal of Abnormal Child Psychology, 44(2), 393-404.

Bauer, D. J., \& Curran, P. J. (2003). Distributional assumptions of growth mixture models: implications for overextraction of latent trajectory classes. Psychological Methods, 8(3), 338-363.

Bauer, D. J., \& Curran, P. J. (2004). The integration of continuous and discrete latent variable models: Potential problems and promising opportunities. Psychological Methods, 9(1), 3-29.

Boyle, M. H., Georgiades, K., Duncan, L., Wang, L., Comeau, J., \& 2014 Ontario Child Health Study Team. (2019). Poverty, neighbourhood antisocial behaviour, and Children's Mental Health Problems: Findings from the 2014 Ontario Child Health Study. The Canadian Journal of Psychiatry, 64(4), 285-293.
Casas, F., \& Gonzalez-Carrasco, M. (2019). Subjective well-being decreasing with age: New research on children over eight. Child Development, 90, 375-394.

Clark, S. L., \& Muthén, B. (2009). Relating latent class analysis results to variables not included in the analysis. Retreived August 30, 2019, from https://www.statmodel.com/download/relatinglca.pdf.

Collins, L., \& Lanza, S. (2010). Latent class and latent transition analysis. Hoboken, NJ: Wiley.

Compton, A. (2016). Examining the longitudinal stability of a dualfactor model of mental health in early elementary school students (Doctoral disertation). Retreived June 20, 2019, from https ://digital.lib.washington.edu/researchworks/bitstream/handl e/1773/37096/Compton_washington_0250E_16391.pdf?seque nce.

De Los Reyes, A., Augenstein, T. M., Wang, M., Thomas, S. A., Drabick, D. A., Burgers, D. E., et al. (2015). The validity of the multi-informant approach to assessing child and adolescent mental health. Psychological Bulletin, 141(4), 858-900.

Deighton, J., Humphrey, N., Belsky, J., Boehnke, J., Vostanis, P., \& Patalay, P. (2018). Longitudinal pathways between mental health difficulties and academic performance during middle childhood and early adolescence. British Journal of Developmental Psychology, 36(1), 110-126.

Deighton, J., Tymms, P., Vostanis, P., Belsky, J., Fonagy, P., Brown, A., et al. (2013). The development of a school-based measure of child mental health. Journal of Psychoeducational Assessment, $31(3), 247-257$

Department of Health and Department for Education. (2015). Future in mind: Promoting, protecting and improving our children and young people's mental health and wellbeing. Retrieved June 28, 2019, from https://www.gov.uk/government/uploads/system/ uploads/attachment_data/file/414024/Childrens_Mental_Healt h.pdf.

Department of Health and Department for Education. (2017). Transforming children and young people's mental health provision: A green paper. Retrieved from https://www.gov.uk/government/ uploads/system/uploads/attachment_data/file/664855/Trans forming_children_and_young_people_s_mental_health_provi sion.pdf.

Diener, E. (1984). Subjective well-being. Psychological Bulletin, 95(3), $542-575$.

Dowdy, E., Furlong, M., Raines, T. C., Bovery, B., Kauffman, B., Kamphaus, R. W., et al. (2015). Enhancing school-based mental health services with a preventive and promotive approach to universal screening for complete mental health. Journal of Educational \& Psychological Consultation, 25(2-3), 178-197.

Dowdy, E., \& Kamphaus, R. W. (2007). A comparison of classification methods for use in predicting school-based outcomes. The California School Psychologist, 12(1), 121-132.

Enders, C. K. (2010). Applied missing data analysis. New York, NY: Guilford Press.

Goodman, R. (1997). The strengths and difficulties questionnaire: A research note. Journal of Child Psychology and Psychiatry, 38(5), 581-586.

Greenberg, M. T., \& Abenavoli, R. (2017). Universal interventions: Fully exploring their impacts and potential to produce populationlevel impacts. Journal of Research on Educational Effectiveness, 10(1), 40-67.

Greenspoon, P. J., \& Saklofske, D. H. (2001). Toward an integration of subjective well-being and psychopathology. Social Indicators Research, 54(1), 81-108.

Hagenaars, J. A., \& McCutcheon, A. L. (2002). Applied latent class analysis. Cambridge: Cambridge University Press.

Heron, J., Croudace, T., Barker, E., \& Tilling, K. (2015). A comparison of approaches for assessing covariate effects in latent class analysis. Longitudinal and Life Course Studies, 6(4), 420-434. 
Hudziak, J. J., Wadsworth, M. E., Heath, A. C., \& Achenbach, T. M. (1999). Latent class analysis of Child Behavior Checklist attention problems. Journal of the American Academy of Child and Adolescent Psychiatry, 38(8), 985-991.

Huebner, E. S., \& Hills, K. J. (2011). Does the positive psychology movement have legs for children in schools? The Journal of Positive Psychology, 6(1), 88-94.

Humphrey, N., Hennessey, A., Ashworth, E., Frearson, K., Black, L., Petersen, K., et al. (2018a). Good Behaviour Game: Evaluation report and executive summary. Retrieved November 1, 2019, from https://pdfs.semanticscholar.org/b2cb/43b8b8f1de6c66f81f34 68820f69b78fcd41.pdf.

Humphrey, N., Hennessey, A., Lendrum, A., Wigelsworth, M., Turner, A., Panayiotou, M., et al. (2018b). The PATHS curriculum for promoting social and emotional well-being among children aged 7-9 years: A cluster RCT. Retrieved October 5, 2019, from https ://www.ncbi.nlm.nih.gov/books/NBK519674/.

Jay, M. A., Mc Grath-Lone, L., \& Gilbert, R. (2019). Data Resource: the National Pupil Database (NPD). International Journal of Population Data Science. https://doi.org/10.23889/ijpds.v4i1.1101.

Keiley, M. K., Bates, J. E., Dodge, K. A., \& Pettit, G. S. (2000). A cross-domain growth analysis: Externalizing and internalizing behaviors during 8 years of childhood. Journal of Abnormal Child Psychology, 28(2), 161-179.

Kelly, R. M., Hills, K. J., Huebner, E., \& McQuillin, S. D. (2012). The longitudinal stability and dynamics of group membership in the dual-factor model of mental health: Psychosocial predictors of mental health. Canadian Journal of School Psychology, 27(4), $337-355$.

Keyes, C. L. M. (2013). Promotion and protection of positive mental health: Towards complete mental health in human development. In I. Boniwell, S. David, \& A. Conley Ayers (Eds.), The Oxford handbook of happiness (pp. 915-925). New York, NY: Oxford University Press.

Kim, E. K., Dowdy, E., Furlong, M. J., \& You, S. (2017). Mental health profiles and quality of life among Korean adolescents. School Psychology International, 38(1), 98-116.

Lima, R., \& Morais, N. (2018). Subjective well-being of children and adolescents: Integrative review. Ciencias Psicológicas, 12(2), 249-260.

Lucas, R. (2016). Subjective well-being in psychology. In M. D. Alder \& M. Fleurbaey (Eds.), The Oxford handbook of well-being and public policy (pp. 403-423). NY: Oxford University Press.

Lyons, M. D., Huebner, E. S., Hills, K. J., \& Shinkareva, S. V. (2012). The dual-factor model of mental health: Further study of the determinants of group differences. Canadian Journal of School Psychology, 27(2), 183-196.

MacCallum, R. C., Zhang, S., Preacher, K. J., \& Rucker, D. D. (2002). On the practice of dichotomization of quantitative variables. Psychological Methods, 7(1), 19-40.

Masyn, K. E. (2013). Latent class analysis and finite mixture modeling. In T. D. Little (Ed.), The Oxford handbook of quantitative methods (pp. 551-611). New York, NY: Oxford University Press.

McElroy, E., Shevlin, M., \& Murphy, J. (2017). Internalizing and externalizing disorders in childhood and adolescence: A latent transition analysis using ALSPAC data. Comprehensive Psychiatry, $75,75-84$.

McLeod, J. D., \& Kaiser, K. (2004). Childhood emotional and behavioral problems and educational attainment. American Sociological Review, 69(5), 636-658.

Monahan, K. C., Oesterle, S., \& Hawkins, J. D. (2010). Predictors and consequences of school connectedness: The case for prevention. The Prevention Researcher, 17(3), 3-7.

Moore, S. A., Dowdy, E., Nylund-Gibson, K., \& Furlong, M. J. (2019a). An empirical approach to complete mental health classification in adolescents. School Mental Health, 11(3), 438-453.
Moore, S. A., Dowdy, E., Nylund-Gibson, K., \& Furlong, M. J. (2019b). A latent transition analysis of the longitudinal stability of dual-factor mental health in adolescence. Journal of School Psychology, 73, 56-73.

Morgan, G. B. (2015). Mixed mode latent class analysis: An examination of fit index performance for classification. Structural Equation Modeling: A Multidisciplinary Journal, 22(1), 76-86.

Muthén, L., \& Muthén, B. (1998-2017). Mplus User's Guide, 8th Edition. Los Angeles, CA: Muthén Muthén

Nylund, K. L., Asparouhov, T., \& Muthén, B. O. (2007). Deciding on the number of classes in latent class analysis and growth mixture modeling: A monte carlo simulation study. Structural Equation Modeling, 14(4), 535-569.

Panayiotou, M., \& Humphrey, N. (2018). Mental health difficulties and academic attainment: Evidence for gender-specific developmental cascades in middle childhood. Development and Psychopathology, 30, 523-538.

Papandrea, K., \& Winefield, H. (2011). It's not just the squeaky wheels that need the oil: Examining teachers' views on the disparity between referral rates for students with internalizing versus externalizing problems. School Mental Health, 3(4), 222-235.

Patalay, P., \& Fitzsimons, E. (2016). Correlates of mental illness and wellbeing in children: Are they the same? Results from the UK Millennium Cohort Study. Journal of the American Academy of Child and Adolescent Psychiatry, 55(9), 771-783.

Patalay, P., Gondek, D., Moltrecht, B., Giese, L., Curtin, C., Stanković, M., et al. (2017). Mental health provision in schools: Approaches and interventions in 10 European countries. Global Mental Health, 4, e10.

Petersen, K. J., Qualter, P., \& Humphrey, N. (2019). The application of latent class analysis for investigating population child mental health: A systematic review. Frontiers in Psychology, 10, 1214.

Polanczyk, G. V., Salum, G. A., Sugaya, L. S., Caye, A., \& Rohde, L. A. (2015). Annual research review: A meta-analysis of the worldwide prevalence of mental disorders in children and adolescents. Journal of Child Psychology and Psychiatry, 56(3), 345-365.

Pryor, L., Strandberg-Larsen, K., Nybo Andersen, A.-M., Hulvej Rod, N., \& Melchior, M. (2019). Trajectories of family poverty and children's mental health: Results from the Danish National Birth Cohort. Social Science and Medicine, 220, 371-378.

Quinlan, D. M., Swain, N., Cameron, C., \& Vella-Brodrick, D. A. (2014). How 'other people matter' in a classroom-based strengths intervention: Exploring interpersonal strategies and classroom outcomes. The Journal of Positive Psychology, 10(1), 77-89.

Ravens-Sieberer, U., Auquier, P., Erhart, M., Gosch, A., Rajmil, L., Bruil, J., et al. (2007). The KIDSCREEN-27 quality of life measure for children and adolescents: Psychometric results from a cross-cultural survey in 13 European countries. Quality of Life Research, 16(8), 1347-1356.

Rebelez Ernst, J. L. (2016). Capturing complete mental health among adolescents: Investigation of covitality latent class typologies (Doctoral dissertation). Retreived September 4, 2019, from https://escholarship.org/content/qt15b8k0rm/qt15b8k0rm.pdf.

Rhemtulla, M., Brosseau-Liard, P. É., \& Savalei, V. (2012). When can categorical variables be treated as continuous? A comparison of robust continuous and categorical SEM estimation methods under suboptimal conditions. Psychological Methods, 17(3), 354-373.

Robitail, S., Ravens-Sieberer, U., Simeoni, M.-C., Rajmil, L., Bruil, J., Power, M., et al. (2007). Testing the structural and cross-cultural validity of the KIDSCREEN-27 quality of life questionnaire. Quality of Life Research, 16(8), 1335-1345.

Rose, T., Lindsey, M. A., Xiao, Y., Finigan-Carr, N. M., \& Joe, S. (2017). Mental health and educational experiences among black 
youth: A latent class analysis. Journal of Youth and Adolescence, 46(11), 2321-2340.

Seligman, M. E., \& Csikszentmihalyi, M. (2000). Special issue on happiness, excellence, and optimal human functioning. American Psychologist, 55(1), 5-183.

Standards and Testing Agency. (2018). Teacher assessment frameworks at the end of key stage 1. Retrieved September 19, 2019, from https://www.gov.uk/government/publications/teacher-assessment -frameworks-at-the-end-of-key-stage-1\#history.

Stone, L. L., Otten, R., Engels, R. C. M. E., Vermulst, A. A., \& Janssens, J. M. A. M. (2010). Psychometric properties of the parent and teacher versions of the strengths and difficulties questionnaire for 4- to 12-year-olds: A review. Clinical Child and Family Psychology Review, 13(3), 254-274.

Suldo, S. M., Hearon, B. V., Bander, B., McCullough, M., Garofano, J., Roth, R. A., et al. (2015). Increasing elementary school students' subjective well-being through a classwide positive psychology intervention: Results of a pilot study. Contemporary School Psychology, 19(4), 300-311.

Suldo, S. M., \& Shaffer, E. J. (2008). Looking beyond psychopathology: The dual-factor model of mental health in youth. School Psychology Review, 37(1), 52-68.

Suldo, S. M., Thalji, A., \& Ferron, J. (2011). Longitudinal academic outcomes predicted by early adolescents' subjective well-being, psychopathology, and mental health status yielded from a dual factor model. The Journal of Positive Psychology, 6(1), 17-30.

Suldo, S. M., Thalji-Raitano, A., Kiefer, S. M., \& Ferron, J. M. (2016). Conceptualizing high school students' mental health through a dual-factor model. School Psychology Review, 45(4), 434-457.

Thalji, A. L. (2012). A dual-factor model of mental health in high school students: Group characteristics and social functioning
(Doctoral dissertation). Retrieved August 10, 2019, from http:// scholarcommons.usf.edu/etd/4239.

The World Health Organisation. (2012). Comprehensive mental health action plan 2013-2020. Retrieved September 1, 2019, from https ://www.who.int/mental_health/action_plan_2013/en/.

Vedi, K., \& Bernard, S. (2012). The mental health needs of children and adolescents with learning disabilities. Current Opinion in Psychiatry, 25(5), 353-358.

Vendlinski, M. K., Javaras, K. N., Van Hulle, C. A., Lemery-Chalfant, K., Maier, R., Davidson, R. J., et al. (2014). Relative influence of genetics and shared environment on child mental health symptoms depends on comorbidity. PLoS ONE, 9(7), e103080.

Wadsworth, M. E., Hudziak, J. J., Heath, A. C., \& Achenbach, T. M. (2001). Latent class analysis of child behavior checklist anxiety/ depression in children and adolescents. Journal of the American Academy of Child and Adolescent Psychiatry, 40(1), 106-114.

Wang, X., Zhang, D., \& Wang, J. (2011). Dual-factor model of mental health: Surpass the traditional mental health model. Psychology, 2(08), 767-772.

Wingate, E. J., Suldo, S. M., \& Peterson, R. K. S. (2018). Monitoring and fostering elementary school students' life satisfaction: A case study. Journal of Applied School Psychology, 34(2), 180-200.

Publisher's Note Springer Nature remains neutral with regard to jurisdictional claims in published maps and institutional affiliations. 